\title{
Formation of ideas about rational nutrition as an element of student's environmental education
}

\author{
Olena Aksonova*, Dmitry Torianik, Victoria Evlash, Dmitry Slivar, and Sergey Gubsky \\ ${ }^{1}$ Kharkiv State University of Food Technology and Trade, 61051 Kharkiv, Ukraine
}

\begin{abstract}
The aim of the work was to form ideas about rational nutrition as an element of ecological education of students by studying and discussing their eating behavior in relation to foods rich in vitamin D using quantitative descriptor-profile analysis. Quantitative descriptor-profile analysis showed that the "poorest" profiles have foods with the highest vitamin D. Thus, foods with a high vitamin D content do not fall into the circle of students' dietary preferences. The results of the survey showed that the most frequently consumed foods for young people are fried potatoes, pizza, dumplings and cheese. Products such as cheese, butter, artificial red caviar, mushrooms (chanterelles) can be successfully used as a base for fortification with vitamin $\mathrm{D}$, since they are positively perceived by students and affordable. It was shown that cheese is the only product that, when surveyed by 59 young people, was included both in the list of 5 most favorite and frequently consumed foods, and was selected from the list of 22 foods offered to the respondents containing significant amounts of vitamin D. The research carried out is the basis for forming students' understanding of the importance of a balanced diet in the context of environmental education.
\end{abstract}

Keywords: vitamin D, rational nutrition, COVID-19.

\section{Introduction}

Nutrition for young people and children is an important issue that requires the attention of the state and family. The state is obliged to create all conditions for the issues of food safety and food security to be resolved. Citizens must have access to quality and safe food; to comprehensive and reliable information about food products that are on store shelves. Environmental education of children, adolescents and youth, in particular, the discussion of healthy nutrition, is also an important aspect of educating generations of citizens who can make a choice towards a healthy lifestyle.

At the present stage, a difficult problem for many countries around the world are the socalled "diseases of civilization", one of the causes of which is the lack of important nutrients in the diet. This fact fully applies to nutrients such as vitamins. A special place among them has recently been given to the problem of vitamin D deficiency. Analysis of preliminary medical statistics [1-4] has shown an important role of this vitamin in the regulation of the immune system in viral infections caused by coronavirus SARS-CoV-2. A

\footnotetext{
*Corresponding author:eaksonova@gmail.com
} 
large number of recent studies suggest that the status of vitamin $\mathrm{D}$ in the blood can determine both the risk of COVID-19 infection and the severity of the disease, as well as affect mortality from COVID-19 [5-11]. Vitamin D deficiency has been shown to be more likely to be observed in patients with laboratory-proven cases of COVID-19 [6]. A population-based study in Israel has shown that low vitamin D levels are associated with a higher likelihood of contracting COVID-19 [8]. A retrospective study conducted in 20 European countries showed that there was a significant correlation between average vitamin D levels and cases of COVID-19 [11]. Studies conducted at the University of Chicago and Switzerland showed that the incidence of COVID-19 was higher in the group of patients with vitamin $\mathrm{D}$ deficiency than in the group with adequate levels of vitamin $\mathrm{D}[12,13]$. The association of the severity and mortality rate from COVID-19 with vitamin D status was noted in $[5,6,14,15]$. It was also shown in [15] that treating severe vitamin D deficiency reduces the risk of high levels of C-reactive protein (CRP levels) in the body. CRP levels are used as a proxy marker of cytokine storm, which is expected to be elevated in severe COVID-19 cases. A study [16] aimed at assessing the nutritional status of patients with COVID-19 and found extreme vitamin D deficiency in $24 \%$ of patients in the confirmed COVID-19 group and $7.3 \%$ in the control group. The authors of [17] cite evidence that vitamin D deficiency is a pandemic, especially in Europe, where $40 \%$ of the population is vitamin $\mathrm{D}$ deficient and $13 \%$ are acutely deficient. In the same work, a link was established between the incidence of COVID-19, vitamin D deficiency and geographical latitude in the countries most severely affected by the pandemic. It has been suggested that this may be due to the fact that vitamin $\mathrm{D}$ is mainly formed under the influence of sunlight, and many European countries are located in latitudes where synthesis under the influence of sunlight is difficult.

The above works allow us to conclude that the optimization of the vitamin status of the population refers to technologies for reducing nutritional and socially significant diseases, and is also a tool for combating the COVID-19 pandemic. Therefore, environmental education and educational work among young people on nutrition and diet correction can become a significant factor that will make the nation healthier.

Proof of the existing problem is, for example, work [18], which summarizes research data on Health effects of dietary risks for the population of 195 countries of the world. The data show that daily consumption of foods that cannot be categorized as rich in nutrients exceeds optimal levels globally. However, in this study and in similar studies in recent years, due attention is not paid to the study of the proportion of foods rich in vitamin D in the diet of the population, while the above studies indicate a direct relationship between the status of vitamin D in the body and the risks from COVID-19.

As already noted, the level of vitamin $\mathrm{D}$ in the body can be raised either by taking vitamin preparations, or by timely and sufficient exposure to the sun, or by consuming foods rich in vitamin D. Ideally, an increase in vitamin D status should occur through a combination of the above. factors. However, the population of countries located at latitudes of $35^{\circ} \mathrm{N}$. natural synthesis of vitamin D by the skin is limited or even completely absent in the winter season. As for the interest of the population in vitamin preparations and food products containing high concentrations of vitamin $\mathrm{D}$, according to the data of the electronic resource Google Trends (trends.google.com), the peak of information requests of users regarding vitamin D occurred in December 2020. At the same time, the demand for information on food products containing vitamin D, against this background, remained stably low. This fact indicates a lack of awareness among the population about the possibility of obtaining this vitamin with food. This situation confirms the relevance of the need for the formation of the population, and especially among young people, of knowledge about healthy nutrition in the framework of environmental education with the dominant factor of usefulness over the factor of taste preference. 
The aim of the work was to form ideas about rational nutrition as an element of ecological education of students by studying and discussing their eating behavior in relation to foods rich in vitamin D using quantitative descriptor-profile analysis according to the ISO 8586: 2012 standard. This analysis allows you to study food profiles and quantify the eating behavior of students by calculating the area of the profiles. The information obtained can be used as a strategy tool for correcting diets in the formation of ideas about rational nutrition as an element of ecological education of students.

\section{Methods}

\subsection{Assessors}

For sensory assessment, first-year students of Kharkiv State University of Food Technology and Trade aged between 17 and 22 years were recruited as tasters of which $78.4 \%$ are women and $21.6 \%$ are men. As a result, the expert group consisted of 10 people. Education and training of students was carried out in accordance with the requirements of the ISO 11035: 1994 standard.

\subsection{Questionnaire/instrument}

In the questionnaires, the respondents had to answer two questions: 1) indicate five food products that they prefer to consume daily; 2) select 5 items from the list of 22 foods containing high concentrations of vitamin D (provided that all foods from the list are easily accessible physically and materially).

Quantitative descriptor-profile analysis was chosen as the method of study. It was used to assess the characteristics of food products, which, based on the literature data, have high levels of vitamin D:

1. "Taste" (A), “Aroma" (B), "Texture" (C) assensore evolution descriptores.

2. "Pleasure" as the pleasure response to foods (E).

3. "Convenience" (D) as universal descriptor, which was understood as the ease of physical access to food sources and the time spent buying and preparing food.

The results obtained were visualized as circular profiles on a five-point scale using levels: 1 - very weak; 2 - weak; 3 - medium; 4 - strong; 5 - very strong.

\subsection{Data analysis}

For ease of comparison of food products, the areas of the polygons formed by the profile diagrams were calculated. The area values were further considered as a summary descriptor "Preference". All constructions and calculations were performed using the program Excel Microsoft Office (Microsoft Inc., USA).

Hierarchical cluster analysis (HCA) is an unsupervised classification approach for analysing multivariate data through which objects are grouped into clusters. This method was performed on standardized data to classify the samples based on the similarities of their sensory data and vitamin D amount data. For this purpose, the data were centered and divided by their standard deviation to ensure that all the elements had equal weight in the results:

$$
\begin{aligned}
\bar{X}_{J}=\sum_{i=1}^{n} \frac{X_{i j}}{n} & \\
s_{j} & =\sqrt{\sum_{i=1}^{n}\left(X_{i j}-\bar{X}_{j}\right)^{2} / n}(2)
\end{aligned}
$$


where $n$ is total samples, $\bar{X}_{j}$ is mean value $X_{i j}$ of $j$-variables and $i$-samples.

The linkage method, which was applied here, was Ward's method. Clusters were calculated using the Euclidian distance. Moreover, the statistical significance of the obtained clusters was tested by various formal and empirical statistical criteria: ratios of agglomeration and cross-tabulation and adjusted $R_{a d j}^{2}[19]$ :

$$
R_{a d j}^{2}=1-\frac{W S S(n-1)}{T W S S(n-k)}
$$

where WSS is within the cluster the sum of squares of distances; TWSS is within sampling the total sum of the squares of distances; $k$ is number of clusters. The HCA analysis was performed by using and Minitab ver. 18 (Minitab Inc., USA).

\subsection{Direct questioning}

Direct questioning was conducted in 59 people aged 17 to 22 years (females $-78.4 \%$, males $-21.6 \%$ between the ages of 17 and 22 ) in order to identify food preferences in the daily diet. In the questionnaires, the respondents had to answer two questions: 1) indicate five food products that they prefer to consume daily; 2) select 5 items from the list of 22 foods containing high concentrations of vitamin D (provided that all foods from the list are easily accessible physically and materially)

\section{Results and discussion}

In a study [20], it was shown that foods low in protein, vitamins and other valuable nutrients tend to be high in fat and have a pronounced sweet or salty taste, attractive appearance and pleasant smell. It should be noted that the typical flavor profile of low nutrient foods (a profile that takes into account taste, color, smell, appearance, convenience, and budget price) makes them attractive to consumers. However, as the authors of the article note, taste preferences and subsequent food choices can change as a result of competent environmental education, especially in childhood, adolescence and youth, when taste preferences play an important role in the choice and consumption of food. Strategies that emphasize the good taste of nutrient-rich foods are especially recommended for those consumers who are more taste-oriented than health-oriented. The flavor profile of low nutrient foods is more consistent than nutrient rich foods. Thus, the analysis of product profiles and the determination of the area occupied by the profile is a tool that will describe the eating behavior of students and offer them strategies for correcting diets in the direction of eating healthy and wholesome food.

The results of descriptor-profile analysis of 22 foods rich in vitamin D are presented in Table 1. From Table 1, it can be seen that cheese has the largest area, and fish oil has the smallest area. Atlantic salmon, humpback salmon, mackerel, and quail eggs also have high area values. Red caviar, mushrooms (chanterelles), and butter have fairly high area values. It is these products that should be paid attention to, as the products most suitable for fortification with vitamin D. Moreover, depending on the product, fortification can be carried out both by the direct introduction of vitamin $\mathrm{D}$ (when it comes to the production of artificial red caviar or butter), or by spontaneous production of this vitamin directly in the product under the influence of ultraviolet radiation (for mushrooms). 
Table 1. Students preferences to foods with vitamin $\mathrm{D}$ amount (in $\mu \mathrm{g} / 100 \mathrm{~g}$ of samples).

\begin{tabular}{|c|c|c|c|c|c|c|c|c|}
\hline Cluster & Food & Amount & $\mathbf{A}$ & B & $\mathbf{C}$ & $\mathbf{E}$ & D & Preference \\
\hline \multirow{8}{*}{ I } & Cheese & 1 & 4.5 & 4.6 & 4.6 & 4.5 & 4.5 & 39.4 \\
\hline & Humpback salmon & 11 & 4.4 & 4.4 & 4.6 & 4.6 & 4.3 & 37.6 \\
\hline & Atlantic salmon & 11 & 4.9 & 4.7 & 4.9 & 4.7 & 5.0 & 37.2 \\
\hline & Mackerel & 16 & 4.5 & 4.1 & 4.5 & 4.5 & 4.4 & 36.4 \\
\hline & Quail egg & 1 & 4.4 & 4.4 & 4.3 & 4.4 & 4.2 & 35.6 \\
\hline & Caviar red & 3 & 4.2 & 4.1 & 4.4 & 4.2 & 4.0 & 33.1 \\
\hline & Chicken egg & 2 & 4.0 & 3.8 & 3.8 & 4.1 & 4.5 & 31.0 \\
\hline & Chanterelles & 5 & 3.9 & 3.9 & 3.8 & 4.2 & 4.0 & 29.5 \\
\hline \multirow{6}{*}{ II } & Flatfish & 3 & 3.7 & 3.5 & 3.6 & 3.6 & 3.9 & 25.5 \\
\hline & Sprats in oil & 21 & 4.1 & 2.7 & 3.4 & 3.8 & 4.4 & 24.7 \\
\hline & Butter & 1 & 3.0 & 3.0 & 3.4 & 3.7 & 4.5 & 24.3 \\
\hline & Caviar black & 8 & 3.5 & 3.3 & 4.2 & 3.7 & 3.1 & 23.7 \\
\hline & Tuna & 6 & 3.6 & 3.5 & 3.6 & 3.6 & 3.1 & 22.6 \\
\hline & Salty fat herring & 30 & 3.1 & 2.5 & 3.5 & 3.4 & 3.7 & 20.0 \\
\hline \multirow{7}{*}{ III } & Dog-salmon & 16 & 2.9 & 2.4 & 3.0 & 3.2 & 2.8 & 15.0 \\
\hline & Pike & 3 & 3.0 & 2.4 & 2.9 & 2.9 & 2.6 & 13.9 \\
\hline & Codfish liver & 100 & 3.0 & 2.6 & 2.3 & 2.4 & 3.1 & 13.5 \\
\hline & Ghee & 2 & 2.4 & 2.3 & 2.5 & 2.8 & 2.8 & 12.4 \\
\hline & River perch & 3 & 2.5 & 2.0 & 2.6 & 2.2 & 2.7 & 11.0 \\
\hline & Goat's milk & 1 & 2.0 & 1.6 & 2.6 & 2.4 & 2.3 & 9.0 \\
\hline & Sea bass & 2 & 2.2 & 1.9 & 2.1 & 2.0 & 2.2 & 8.2 \\
\hline IV & Fish Oil & 250 & 1.1 & 0.9 & 1.5 & 1.2 & 2.0 & 3.7 \\
\hline
\end{tabular}

Chicken egg and cheese were chosen as the leading products in terms of convenience. This is not surprising, because these products do not require complex technological processing and are easily available in retail chains. If it was necessary to choose products from the list (Table 1), the respondents chose: cheese (mentioned in $81 \%$ of the 
questionnaires), mackerel ( $72 \%$ of the questionnaires), chicken egg $(59 \%$ of the questionnaires), pink salmon (50\% of the questionnaires). Salmon, red caviar and sprats are mentioned in about $40 \%$ of the questionnaires. Thus, we can conclude that young people between the ages of 17 and 22 do not think about the usefulness of the products they consume on a daily basis. However, with proper environmental education, the diets of young people can be adjusted towards healthier foods. It should be noted that hard cheese is included in both preference lists. Young people are ready to use it daily and without preliminary conversations about the usefulness of certain types of products.

Comparison of the profiles shows that the visual appeal of the product and the enjoyment of food are of great importance for young people. There is also some correlation with the theory that the most nutrient-rich foods have the poorest profiles with the smallest area. The direct questioning showed that the most popular product among students is fried potatoes (mentioned in 53\% of the questionnaires), in second place with the same number of points are dumplings, pizza and cheese (41\% of the questionnaires). And in third place are pastry, okroshka (a traditional Ukrainian dish) and red caviar (18\%). The rest of the products were mentioned in less than $10 \%$ of the questionnaires. In the list of taste preferences, there are practically no fruits, vegetables, chicken eggs, sour milk and dairy products, as well as fish and seafood. The HCA searches for patterns of similarity (clusters) either between objects of a certain study or between the features describing objects. Thus, the HCA is a typical unsupervised method for exploratory data analysis that enables more reliable data interpretation [21].

Figure 1 presents the dendrogram of the hierarchical cluster analysis of food samples with vitamin D according to the Vitamin D amount and Preference as their variables. The variable "Vitamin D amount" in this case is actually equivalent to the descriptor "Usefulness", while the variable "Preference" is associated with the variable "Attractiveness". The generated dendrogram shows four clusters with a different number of samples from 1 to 8 (Table 1), where the clusters are mainly linked by decreased distance between samples. To select the number of clusters, we used the procedure described in [19]. First, the selection of four clusters was based on analyzing theagglomerating index as the criterion for identifying thenumber of clusters. Secondary, themain criterion was the formal statistical indicator $R_{a d j}^{2}$, calculated using the formula (3).The consistent rise in the number ofclusters naturally improves this indicator. However, preliminary calculationshows that its noticeable change occurs up to 4 clusters.Further increase in the cluster quantity changes $R_{a d j}^{2}$ insignificantlyonly.

The resulting clusters actually reflect the correlation "Usefulness" vs "Choice" of the studied foodstuffs containing vitamin D. A retrospective review of the obtained clustering allows us to highlight some facts:

1) distribution of 22 food products by clusters was obtained in the form of a ratio of 8 : 6: 7: 1;

2) foods containing the highest amount of vitamin D are in clusters $2-4$, i.e. are less attractive to students;

3) the most useful product Fish Oil forms a separate cluster with the least Preferance;

4)the representation of cluster elements in three-dimensional space Amount D vs Convenience vs Aroma and Amount D vs Pleasure vs Texture (Fig. 2) confirms the idea that the visual attractiveness of the product and the pleasure of eating are more important for young people than its usefulness. 


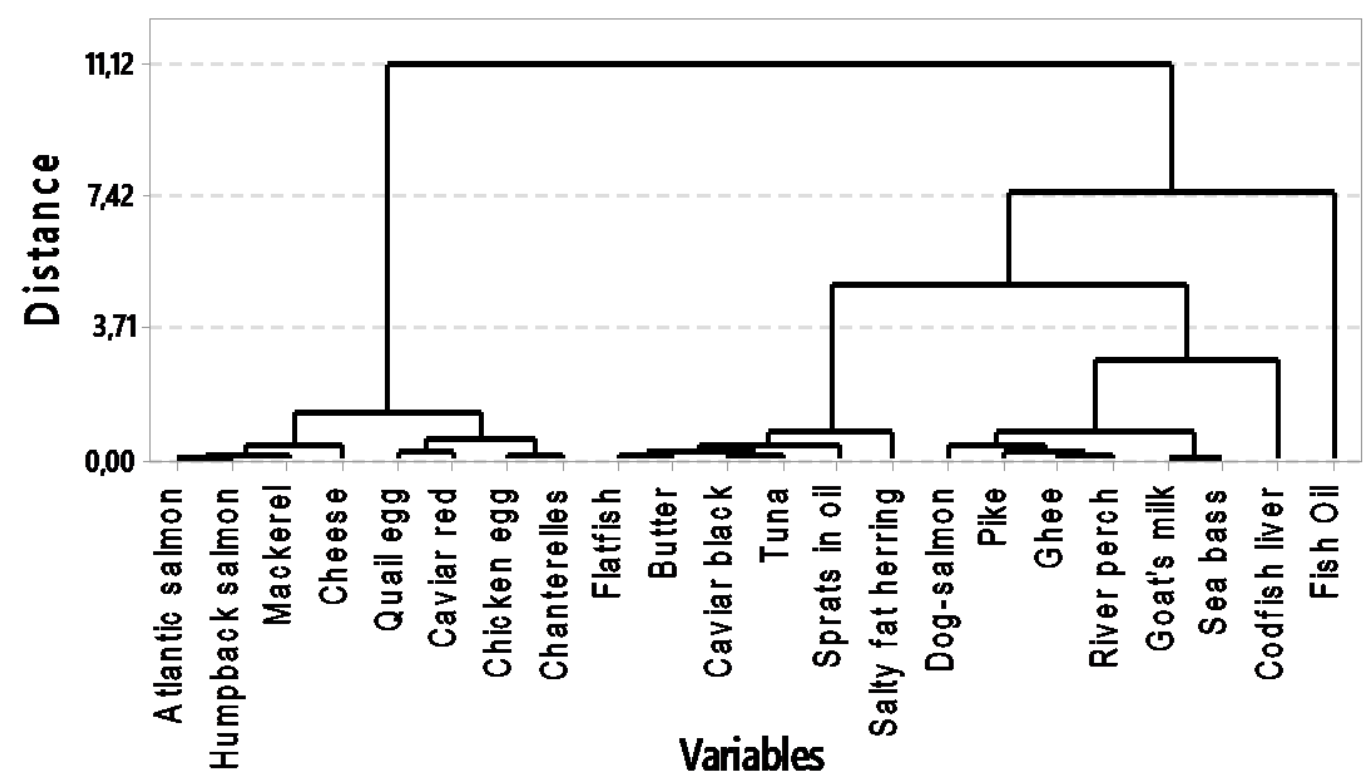

Fig. 1. Hierarchical cluster analysis dendrogram with four distinguished D vitamin-like foods clusters

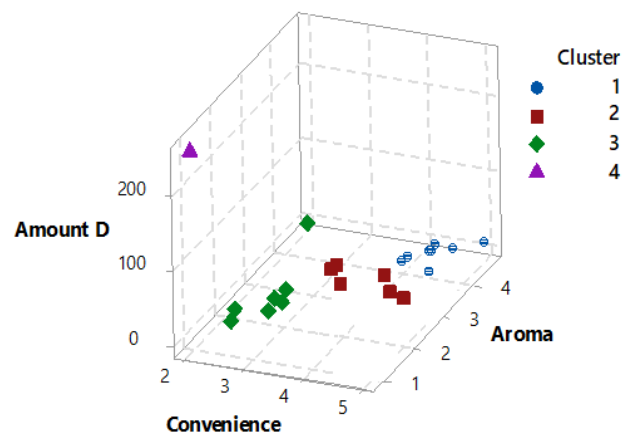

a

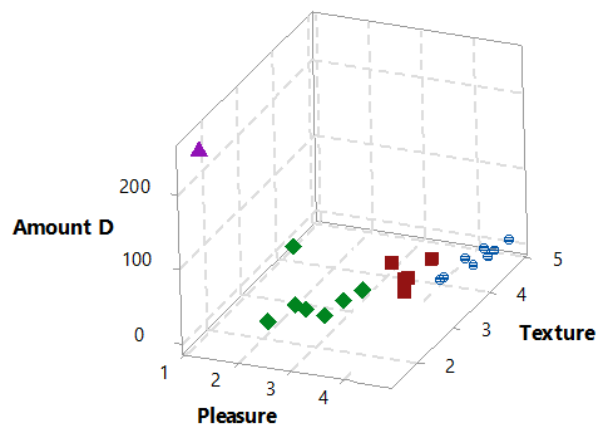

b

Fig. 2. 3D Scatterplot of Amount D vs Convenience vs Aroma (a) and Amount D vs Pleasure vs Texture (b)

\section{Conclusion}

Quantitative descriptor-profile analysis showed that the "poorest" profiles have foods with the highest vitamin D. It can also be seen that cheese has the largest area of the diagram. Thus, we can conclude that foods with a high content of vitamin D do not fall into the circle of students' food preferences. At the same time, foods with the greatest availability are often limited by their high price (Atlantic salmon, humpback salmon).

The results of the direct questioning show that the most frequently consumed and "favorite" products for young people are fried potatoes, pizza, dumplings and cheese. The studies carried out also suggest that products such as cheese, butter, artificial red caviar, mushrooms (chanterelles) can be successfully used as a base for fortification with vitamin D. Since these products are positively perceived by students and are affordable. It should 
also be noted that cheese is the only product that, when surveyed by 59 young people, was included both in the list of 5 most favorite and frequently consumed foods, and was selected from the list of 22 foods offered to respondents containing significant amounts of vitamin D.

The conducted research became the basis for the formation of students' understanding of the importance of a balanced diet in the context of environmental education.

\section{References}

1. M. Pereira, D.A. Dantas, A.L. Galvão, et al., Crit. Rev. Food Sci. Nutr. Nov 4, 1 (2020)

2. K. Ye, F. Tang, X. Liao, et al., J. Am. Coll. Nutr. Oct 13, 1 (2020)

3. N. Vyas, S.J. Kurian, D. Bagchi, et al., J. Am. Coll. Nutr.Sep 1, 1 (2020)

4. H. Yisak, A. Ewunetei, B. Kefale, et al., Risk ManagHealthc Policy,7, 31(2021)

5. G.E. Carpagnano, V. Di Lecce, V.N. Quaranta, et al. J Endocrinol Invest. 44, 765 (2020)

6. A. Mendy, S. Apewokin, A.A. Wells, et al., Factors Associated with Hospitalization and Disease Severity in a Racially and Ethnically Diverse Population of COVID-19 Patients, in medRxiv (2020)

7. A. Daneshkhah, A. Eshein, H. Subramanian, et al., The role of vitamin D in suppressing cytokine storm in COVID-19 patients and associated mortality, in medRxiv (2020)

8. E. Merzon, D. Tworowski, A. Gorohovski, et al, FEBS J. 287, 17 (2020)

9. A. Fasano, E. Cereda, M. Barichella, et al. Movement Disorders, 35, 7 (2020)

10. P. Raharusun, S. Priambada, C.Budiarti, et al., Patterns of COVID-19 Mortality and Vitamin D: An Indonesian Study, in SSRN Electronic Journal(2020)

11. N. Ali,J Infect Public Health,13, 10 (2020)

12. D.O. Meltzer, T.J. Best,H. Zhang, et al, Association of Vitamin D Deficiency and Treatment with COVID-19 Incidence, in medRxiv(2020)

13. A. D'Avolio, V. Avataneo, A. Manca, et al, Nutrients, 12, 5 (2020)

14. M. Entrenas Castillo, L.M. Entrenas Costa, J.M. Vaquero Barrios, et al, J Steroid Biochem. Mol. Biol., 203, 105751 (2020)

15. A. Daneshkhah, A. Eshein, H. Subramanian, et al, The Possible Role of Vitamin D in Suppressing Cytokine Storm and Associated Mortality in COVID-19 Patients, in medRxiv (2020)

16. J.H. Im,Y.S. Je, J. Baek, M.-Y. Chung, et al, Int. J. Infect. Dis., 100 ( 2020)

17. M. Kara, T. Ekiz, V. Ricci, Ö Kara, Br. J.Nutr.124,736 (2020)

18. GBD 2017 Causes of Death Collaborators,Lancet, 393, 1958(2019)

19. A. Goralchuk, S. Gubsky, S. Omel'chenko, et al, Eur. Food Res. Technol.,246, 1955 (2020)

20. D.G. Liem, C.G. Russell, Frontiers in Nutrition, 6, 174 (2019)

21. K. Niranjan, S.F.J Silva, Bubbles in Foods: Creating Structureout of Thin Air, in Food engineering: integrated approaches (Springer, New York, 2008) 